\title{
ARTÍCULOS
}

\section{¿QUÉ ESTÁ PASANDO CON LOS ESTUDIOS UNIVERSITARIOS DE BIBLIOTECONOMÍA Y DOCUMENTACIÓN EN ESPAÑA?}

\author{
Virginia Ortiz-Repiso, Javier Calzada-Prado e Ivett M. Aportela-Rodríguez
}

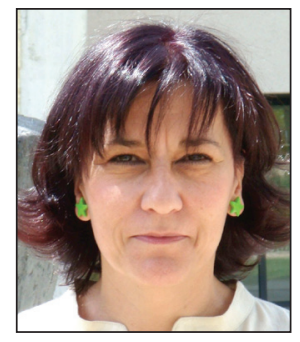

Virginia Ortiz-Repiso es desde 1996 profesora titular del Departamento de Biblioteconomía y Documentación de la Universidad Carlos III de Madrid, del que es actualmente su directora. Es presidenta de la Red de Centros y Departamentos de Información y Documentación (RUID) desde 2011 y representante de España en el Capítulo Europeo de ASIS\&T (American Society for Information Science and Technology) desde 2012. Los temas principales de sus trabajos están vinculados con la gestión de recursos tecnológicos en bibliotecas digitales, la recuperación de información y la evaluación de sedes web.

http://orcid.org/0000-0002-4509-4464

virginia@bib.uc3m.es

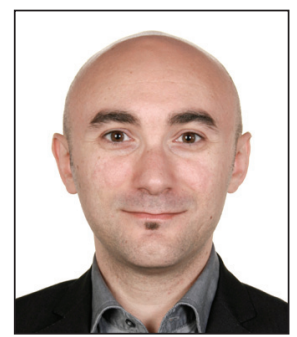

Javier Calzada-Prado es profesor del Departamento de Biblioteconomía y Documentación de la Universidad Carlos III de Madrid. Doctor en documentación por dicha universidad, su actividad docente e investigadora se centra en la contribución de los servicios de apoyo al aprendizaje y la investigación a la innovación y la construcción de conocimiento. En este sentido, ha realizado y colaborado en diversas publicaciones sobre competencias informacionales, diseño de contenidos educativos y la organización y representación de recursos en repositorios y bibliotecas digitales educativas

http://orcid.org/0000-0002-0806-5242

fcalzada@bib.uc3m.es

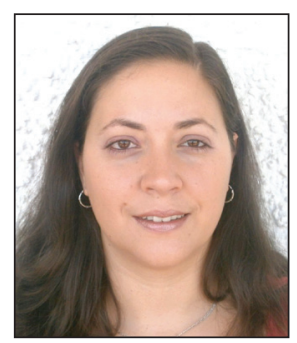

Ivett M. Aportela-Rodríguez es personal investigador en formación del Departamento de Biblioteconomía y Documentación de la Universidad Carlos III de Madrid y cursa sus estudios de doctorado en dicha universidad. Entre sus líneas de investigación destacan la inteligencia competitiva, los servicios de información en parques científicos y tecnológicos, y las bibliotecas universitarias. Anteriormente estuvo a cargo de un grupo de gestión de información y conocimiento en una consultoría de información.

http://orcid.org/0000-0002-9570-5999

iaportel@bib.uc3m.es

Departamento de Biblioteconomía y Documentación Universidad Carlos III de Madrid

C/ Madrid, 126. 28903 Getafe (Madrid), España

\section{Resumen}

Se analiza la evolución y situación actual de los estudios de biblioteconomía y documentación en España. Se toman como referencia los datos aportados por las universidades miembros de la Red de Centros y Departamentos de Información y Documentación $(R U I D)$ y por fuentes oficiales como el Instituto Nacional de Estadística y el Ministerio de Educación, Cultura y Deportes. Se describe la situación actual de las enseñanzas en extinción, el grado, los masters y los doctorados. Entre otros aspectos, se observa un retroceso en la demanda del grado y cierto aumento en la de los masters y doctorados. Se aportan finalmente algunas reflexiones sobre los retos a los que se enfrentan actualmente estas enseñanzas, así como posibles estrategias que puedan seguirse para fortalecer la disciplina y la profesión en su conjunto.

\section{Palabras clave}

Información y documentación, Biblioteconomía y documentación, Grados universitarios, Masters, Educación universitaria, Oferta formativa, España, Red de Centros y Departamentos de Información y Documentación (RUID), Estadísticas.

\section{Title: What is happening to library and information studies in Spanish universities?}

\section{Abstract}

This article aims to provide an overview of the current state of library and information science (LIS) education in Spanish universities. Data have been gathered from official statistical sources (Spanish Ministry of Education, Culture and Sports 
and National Institute of Statistics) and universities' annual reports, in order to complement data provided by members of the Spanish Network of Information and Documentation University Centers and Departments (RUID). Among other issues, a general decrease in the demand of LIS undergraduate studies and a slight increase in postgraduate studies are observed. Finally, the article presents several reflections on the challenges that these studies are currently facing, and suggests strategies that may be followed to strengthen not only LIS education but also the profession as a whole in this country.

\section{Keywords}

Library and information science, Universities, Spain, Spanish Network of Information and Documentation University Centres and Departments (RUID), LIS education, Statistics.

Ortiz-Repiso, Virginia; Calzada-Prado, Javier; Aportela-Rodríguez, Ivett M. (2013). “¿Qué está pasando con los estudios universitarios de biblioteconomía y documentación en España?”. El profesional de la información, noviembre-diciembre, v. 22, n. 6, pp. 505-514.

http://dx.doi.org/10.3145/epi.2013.nov.02

\section{Introducción}

El origen de este trabajo tiene lugar en la reunión de la Red de Centros y Departamentos de Información y Documentación (RUID) celebrada el 1 de febrero de 2013 en la Universidad Carlos III de Madrid. En ella, los representantes de las universidades participantes se propusieron analizar la situación actual de los estudios de información y documentación en España, a fin de reflexionar y debatir en torno a las posibles medidas que se deben tomar para fortalecerlos.

Los datos de partida del presente artículo fueron los aportados por las universidades respecto a:

- oferta de titulaciones;

- matrícula y número de egresados en el último quinquenio;

- tasas de abandono;

- participación en otras titulaciones;

- fortalezas y debilidades percibidas.

Dado que algunos centros no enviaron datos o estos eran insuficientes, se fueron completando con aquellos disponibles en las siguientes fuentes oficiales: INE, Ministerio de Educación, Cultura y Deporte, comunidades autónomas, memorias académicas y servicios estadísticos de las propias universidades.

\section{La formación en información y documen-} tación en España responde inicialmente a la necesidad de dotar principalmente las bibliotecas públicas

EL objetivo fundamental es realizar una radiografía de los estudios universitarios de biblioteconomía y documentación en España en sus tres ciclos (grados, masters y doctorados) que permita reflexionar sobre los retos a los que se enfrentan estas enseñanzas, así como apuntar las estrategias que pueden seguirse para fortalecer la disciplina y la profesión.

El análisis de los estudios del área en nuestro país ha sido abordado anteriormente en diversos trabajos, entre los que cabe destacar a Abadal (1993), Abadal y Miralpeix (1999), Delgado-López-Cózar (2003; 2007), Delgado-López-Cózar y De-la-Moneda-Corrochano (2008; 2010), García-Marco (2007, 2008), De-la-Moneda-Corrochano (2012) y TejadaArtigas (2013). El presente trabajo es, obviamente, deudor y continuador de sus aportaciones al tiempo que trata de ofrecer nuevos datos y perspectivas.

\section{Centros en los que actualmente se imparten los estudios}

Los estudios del área de información y documentación, en alguno o en todos sus niveles y modalidades, se imparten actualmente en 16 universidades:

- Universidad Carlos III de Madrid (UC3M);

- Universidad Complutense de Madrid (UCM);

- Universidad de Alcalá de Henares (UAH);

- Universidad de Extremadura (UEX);

- Universidad de Granada (UGR);

- Universidad de León (Unileon);

- Universidad de Murcia (UM);

- Universidad de Salamanca (USAL);

- Universidad de Valencia (UV);

- Universidad de Zaragoza (Unizar);

- Universidad Politécnica de Valencia (UPV);

- Universidade de A Coruña (UDC);

- Universitat Autònoma de Barcelona (UAB) (tiene adscrita la Escuela Superior de Archivística y Gestión de Documentos, Esaged),

- Universitat de Barcelona (UB);

- Universitat Oberta de Catalunya (UOC);

- Universitat Pompeu Fabra (UPF).

En algunas de ellas, dada la transversalidad de la especialidad, la formación en materias relacionadas con la disciplina se ha extendido a otros grados y posgrados, siendo en algunos casos la única docencia impartida por los profesores del área.

\section{El pasado más reciente en extinción: diplomatura y licenciatura}

Desde la publicación de los reales decretos que establecieron la estructura de las enseñanzas universitarias oficiales para el EEES, las diplomaturas y licenciaturas del área se han ido extinguiendo y/o transformándose. En el curso $2010 / 2011$ ya no se ofrecieron plazas de nuevo ingreso y en septiembre de 2015 deberán extinguirse todas. 


\section{Diplomatura}

La formación vinculada al área de información y documentación en España responde inicialmente a la necesidad de dotar a la Administración de profesionales con formación específica, abocados principalmente a las bibliotecas públicas (Abadal, 1993). En efecto, el período de expansión de las bibliotecas públicas que tuvo lugar en la década de 1980 y, sobre todo, en la de 1990 (Hernández, 2001) se tradujo en un aumento proporcional en la demanda de la diplomatura en biblioteconomía y documentación (DBD). Como puede observarse en la figura 1 , las líneas correspondientes a estudiantes matriculados totales y de nuevo ingreso describen una tendencia ascendente continua entre los años 1982 y 1999, con el máximo histórico de nuevos ingresos en el curso 1997/1998 (1.561 alumnos) y el de matriculados totales en el curso 1999/2000 (4.859 alumnos), con una zona valle de 1993 a 1998 achacable, quizá, a la crisis económica de 1993-1997.

En 1999, Abadal y Miralpeix comentaban los altibajos que la formación del área había experimentado en Estados Unidos desde los años 70, indicando que "aquí no se han producido altibajos (...) sino que, más bien, la formación de profesionales la podemos representar, hasta el presente, como una línea en continuo crecimiento. No sólo no se ha cerrado ninguna escuela, sino que se está viviendo aún un proceso de expansión continuada que no parece remitir, al menos por el momento".

La situación, a partir de entonces, cambió radicalmente. Delgado-López-Cózar y De-la-Moneda-Corrochano (2008; 2010) describían la situación de los primeros años del siglo XXI como de "estancamiento" y, la de los años siguientes (hasta 2010, fecha del último de sus estudios consultados) de "declive manifiesto", un hecho que también habían señalado en sus trabajos anteriores (Delgado-López-Cózar, 2003; 2007). La figura 1 muestra cómo, efectivamente, el número de estudiantes matriculados en la diplomatura en biblioteconomía y documentación experimentó un descenso continuo en el conjunto de universidades hasta los últimos años de su impartición.

Entre 1982 y 2010 la Diplomatura recibió 14.893 estudiantes de nuevo ingreso en todo el país, de los que se graduaron el 73,7\%. (INE, 2012; 2013; Abadal; Miralpeix, 1999). Sin embargo, la tasa de graduación para el período comprendido entre los cursos 1995/1996 y 2010/2011 varía de manera significativa entre las distintas universidades desde el 91,1\% en la UC3M hasta el $44,6 \%$ de la UV (figura 2 ).

\section{Licenciatura}

A finales de los 80 , la creciente demanda de la diplomatura justificó la necesidad de dotar a los estudios de un segundo ciclo que permitiera a los diplomados completar su formación. El RD 912/1992 establece el título de licenciado en documentación (LD), que iniciaría su andadura en el curso académico 1994/1995 en la UAH, UC3M, UGR y USAL. El rápido ascenso del número de estudiantes de nuevo ingreso que recibe el título (figura 1), impulsado por el creciente número de diplomados (4.077 en 1994) llega a su máximo histórico en el curso 1999/2000 (1.575 alumnos), consiguiendo superar en demanda a la diplomatura en la mayoría de los siguientes cursos, aunque acompañando a ésta en su descenso. En este sentido, De-la-Moneda-Corrochano (2012) señala que la explicación de este hecho podría estar relacionada con la necesidad de los ya diplomados de acceder a una titulación superior, y adicionalmente, por ser una vía para la obtención de un segundo ciclo para quienes ya se les había extinguido esta posibilidad en su área de conocimiento.

En su concepción, el GID trató de dar respuesta tanto a la caída en la demanda observada en las antiguas titulaciones como a las nuevas condiciones del mercado laboral

Hasta el curso 2010/2011, la licenciatura tuvo 12.373 matrículas de nuevo ingreso y 7.401 titulados, con lo que la tasa provisional de graduación es del 59,8\% (INE, 2012; 2013). Varias universidades aún tienen estudiantes matriculados en el curso 2012/2013, por lo que es de esperar que el número de licenciados aumente ligeramente hasta 2015 y, con ello, la tasa final de graduación.

En la distribución por universidades para el período comprendido entre los cursos 1995/1996 y 2010/2011 (figura $3)$, la tasa de graduación va del $93,6 \%$ de la $U A H$ al $41,7 \%$ de la UOC.

\section{Y llegó Bolonia: grado en información y documentación}

El diseño del grado en información y documentación (GID) se perfiló en el Libro blanco de la titulación (Aneca, 2004), resultado del trabajo de una comisión coordinada por la $U B$. 


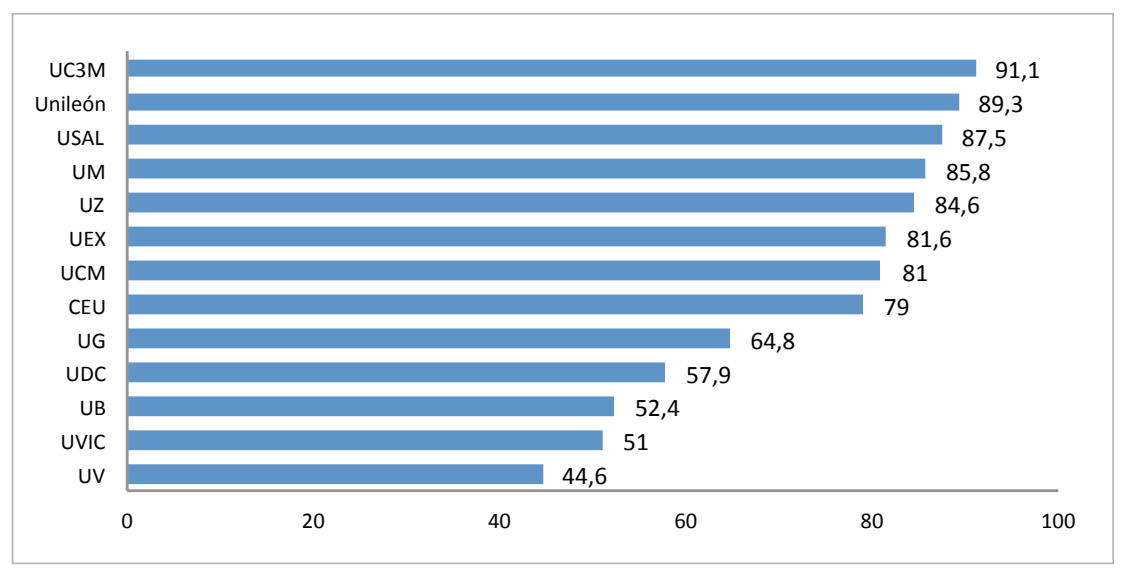

Figura 2. Tasa de graduación de la diplomatura en biblioteconomía y documentación por universidades (1995/1996 a 2010/2011).

Fuente: INE (2012, 2013)

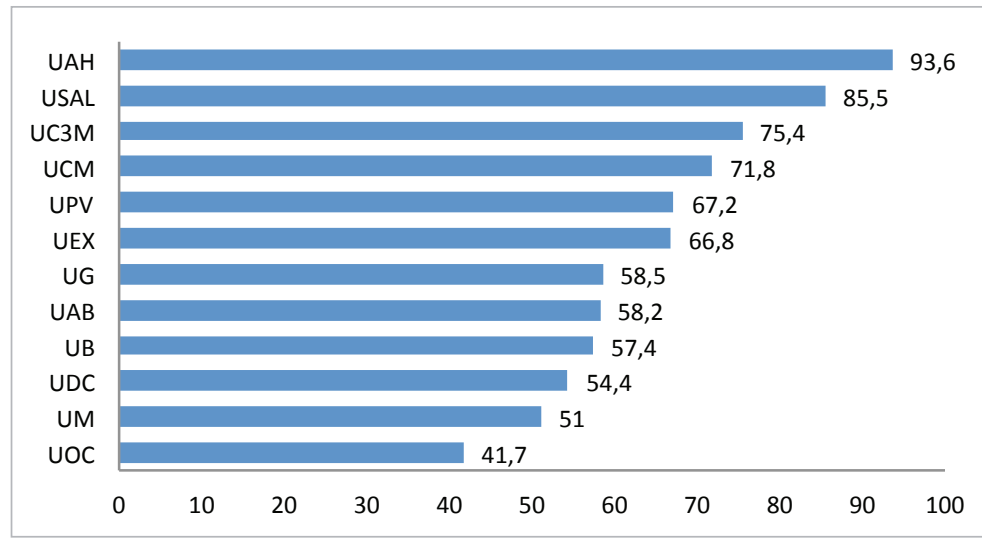

Figura 3. Tasa de graduación de la licenciatura en documentación por universidades (1995/1996 a 2010/2011).

Fuente: INE (2012; 2013).

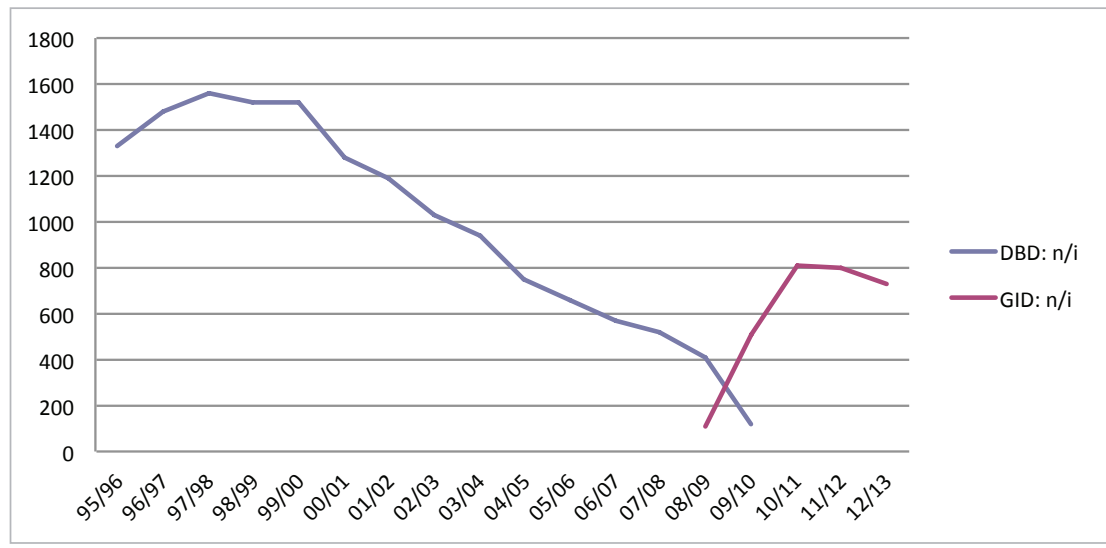

Figura 4. Estudiantes de nuevo ingreso en la diplomatura en biblioteconomía y documentación y en el grado en información y documentación (1995/1996 a 2012/2013).

Fuente: INE (2012; 2013) y universidades

La publicación de los Reales decretos 55/2005 y 1393/2007 pondría en marcha la implantación de este título, que comenzaría a impartirse en 2008 en la UC3M, USAL y Unizar.

En su concepción, el GID trató de dar respuesta tanto a la caída en la demanda observada en las antiguas titulaciones como a las nuevas condiciones del mercado laboral, caracterizado por la disminución del volumen de empleo público y el progresivo aumento de la oferta procedente del sector privado (Aneca, 2004; Montes-López, 1995; Tejada-Artigas; Moreiro-González, 2003; Abadal; Borrego; Serra-Pérez, 2012). Para ello, ha buscado conectar con los modelos euro- peos, tomando como principal referente el euroreferencial de ECIA (European Council of Information Associations) (Euroguide LIS: the guide to competencies for European professionals in library and information services, 1999). Para García-Marco (2009), los grados en información y documentación constituyen un avance positivo en la línea apuntada por la iniciativa ischools estadounidense (hoy ya de ámbito internacional). Morales-Sanabria (2012) resume la estrategia: "el aspecto decisivo pasa por abordar un espectro de competencias y salidas profesionales más amplio y ofrecer paralelamente las especialidades más acordes a sus fortalezas y a las necesidades de su entorno específico".

El análisis de la demanda del GID (figura 4) sugiere que éste ha logrado modificar ligeramente la tendencia de la diplomatura, llevándonos en los cursos 2010/2011 y 2011/2012 a tasas de demanda similares a las del curso 2004/2005, como apuntaba De-la-Moneda-Corrochano (2012).

Sin embargo, entre los cursos 2011/2012 y 2012/2013 se aprecia un retroceso en la demanda global del GID de un 7,9\%. Las causas de este retroceso, cuya evolución habrá de ser estudiada en profundidad en posteriores trabajos, deben buscarse en diversos factores, tales como la capacidad de atracción de la titulación (la cultura en este ámbito es escasa o inexistente), la situación socioeconómica, el número de becas disponibles o los precios públicos de matrícula, entre otros. En este último curso, la mayor parte de las comunidades autónomas han elevado sus tasas universitarias (tabla 1), aunque de manera desigual en el conjunto del territorio.

Sería fácil y tentador, aunque, quizá, no del todo serio, establecer una relación causa/efecto: aumento de tasas/descenso de matrícula. Se requiere un análisis mucho más amplio. Pero qué duda cabe de que la subida de tasas, la situación económica del país, nuestra capacidad de atracción..., inciden negativamente en la elección de los estudios. Aun así, se dan casos singulares. Por ejemplo, se puede observar que en la $U B$ han aumentado un $66,7 \%$ las tasas y la matrícula de nuevo ingreso ha bajado un 29,2\%. En Aragón, las tasas suben un 3,6\% y se reduce la matrícula un $31,6 \%$. Mientras que en Madrid han subido las tasas un $38,1 \%$ y la matricula ha aumentado un $3,3 \%$.

El coste de la matrícula del GID en las universidades públicas (tabla 2), según precios del curso 2012/2013, oscila entre los 2.364 y los $6.064,80$ euros, coste que se incrementa en 
Tabla 1. Comparación de la matrícula de nuevo ingreso con la variación de tasas públicas (2010/2011 a 2012/2013).

Fuente: MEC (2013a) y sedes web de las universidades

\begin{tabular}{|c|c|c|c|c|c|}
\hline & 08/09 & $09 / 10$ & $10 / 11$ & $11 / 12$ & $12 / 13$ \\
\hline \multicolumn{6}{|l|}{ Andalucía } \\
\hline UGR (alumnos $\mathrm{n} / \mathrm{i}$ - var.) & & & 68 & $93(36 \%)$ & $115(23,6 \%)$ \\
\hline Var. tasas comunidad & & & $1,7 \%$ & $4,3 \%$ & $2,4 \%$ \\
\hline \multicolumn{6}{|l|}{ Aragón } \\
\hline Unizar (alumnos n/i - var.) & 25 & $26(4 \%)$ & $16(-38,5 \%)$ & $38(137,5 \%)$ & $26(-31,6 \%)$ \\
\hline Var. tasas comunidad & & $2 \%$ & $2 \%$ & $3,6 \%$ & $3,6 \%$ \\
\hline \multicolumn{6}{|l|}{ Castilla y León } \\
\hline Unileon (alumnos n/i -var.) & & & 14 & $20(42,8 \%)$ & $15(-25 \%)$ \\
\hline USAL (alumnos n/i - var.) & 12 & $14(16,6 \%)$ & $40(185,7 \%)$ & $41(2,5 \%)$ & $32(-21,95 \%)$ \\
\hline Total comunidad (var. n/i) & & & $54(285,7 \%)$ & $61(13 \%)$ & $47(-23 \%)$ \\
\hline Var. tasas comunidad & & $1,5 \%$ & $s / d$ & $7,6 \%$ & $41,9 \%$ \\
\hline \multicolumn{6}{|l|}{ Cataluña } \\
\hline UB (alumnos n/i - var.) & & 94 & $120(27,6 \%)$ & $106(-11,7 \%)$ & $75(-29,2 \%)$ \\
\hline Var. tasas comunidad & & & $5,1 \%$ & $7,6 \%$ & $66,7 \%$ \\
\hline \multicolumn{6}{|l|}{ Extremadura } \\
\hline UEX (alumnos n/i -var.) & & & 49 & $57(16,3 \%)$ & $56(-1,7 \%)$ \\
\hline Var. tasas comunidad & & & $1,5 \%$ & $3,7 \%$ & $1,9 \%$ \\
\hline \multicolumn{6}{|l|}{ Galicia } \\
\hline UDC (alumnos $\mathrm{n} / \mathrm{i}$ - var.) & & 10 & $9(-10 \%)$ & $12(33,3 \%)$ & $10(-16,6 \%)$ \\
\hline Var. tasas comunidad & & & $1,5 \%$ & $3,6 \%$ & $0 \%$ \\
\hline \multicolumn{6}{|l|}{ Madrid } \\
\hline UC3M (alumnos $n / i$ - var.) & 72 & $76(5,5 \%)$ & $90(18,42 \%)$ & $81(10 \%)$ & $83(2,46 \%)$ \\
\hline UCM (alumnos n/i - var.) & & 73 & $99(26,2 \%)$ & $99(0 \%)$ & $103(4 \%)$ \\
\hline Total Comunidad (var. n/i) & & & $26,8 \%$ & $-4,8 \%$ & $3,3 \%$ \\
\hline Var. tasas comunidad & & $1,4 \%$ & $4 \%$ & $5 \%$ & $38,1 \%$ \\
\hline \multicolumn{6}{|l|}{ Murcia } \\
\hline UM (alumnos n/i - var.) & & & 56 & $42(-25 \%)$ & $52(23,8 \%)$ \\
\hline Var. tasas comunidad & & & $0 \%$ & $5 \%$ & $11,8 \%$ \\
\hline \multicolumn{6}{|l|}{ Valencia } \\
\hline UV (alumnos n/i - var.) & & 54 & $64(18,5 \%)$ & $51(-20,3 \%)$ & $51(0 \%)$ \\
\hline Var. tasas comunidad & & & $4 \%$ & $7,5 \%$ & $33,3 \%$ \\
\hline
\end{tabular}

$\mathrm{n} / \mathrm{i}=$ nuevo ingreso; $\mathrm{s} / \mathrm{d}=\sin$ datos

las segundas y terceras matrículas. En este sentido merecen una mención especial los títulos que se ofertan en modalidad semipresencial u online, puesto que compiten fuera de las tradicionales áreas geográficas de influencia de cada universidad. Por su parte, el coste de la matrícula en el GID de una universidad privada puede ascender hasta los 12.000 euros (MEC, 2013a).

\section{Vamos al segundo ciclo: masters}

A lo largo de los últimos años, los estudios de posgrado han experimentado varias reformas. Como complemento a la nueva estructura general definida en el RD55/2005, el $R D 56 / 2005$ reguló de manera específica los estudios de posgrado hasta la entrada en vigor del $R D 1393 / 2007$, actual marco de referencia. Conforme a este último, los masters, que pueden tener de 60 a 120 créditos, "tienen como finalidad la adquisición por el estudiante de una formación avanzada, de carácter especializado o multidisciplinar, orientada a la especialización académica o profesional, o bien a pro- mover la iniciación en tareas investigadoras" (artículo 10). La oferta de titulaciones de segundo ciclo con vinculación con el área es amplia. Se han registrado 53 títulos, de los cuales $26(49,1 \%)$ son masters oficiales y $27(50,9 \%)$ son títulos propios (tabla 3).

Desde el punto de vista de su cobertura temática (figura 5), observamos que predominan los títulos generalistas con un enfoque "digital" (biblioteconomía, documentación y archivística) así como los relativos a information management (gestión de información, gestión de contenidos, gestión del conocimiento, social media, etc.). También tienen una destacada representación los que se ocupan de museología, archivística y biblioteconomía, mientras que la biblioteconomía escolar y otros masters de temática especializada como documentación médica o documentación musical representan el menor porcentaje.

Respecto a su vinculación directa al área de información y documentación por su adscripción a departamentos, cen- 
Tabla 2: Precios GID de primera matrícula completa (cursos 2011/2012 y 2012/2013).

Fuente: MEC (2013a)

\begin{tabular}{|c|c|c|c|c|c|c|}
\hline \multirow{2}{*}{ Universidad } & \multirow{2}{*}{ Modalidad } & \multirow{2}{*}{ Créditos } & \multicolumn{2}{|c|}{$2011 / 2012$} & \multicolumn{2}{|c|}{$2012 / 2013$} \\
\hline & & & $€ /$ crédito & $€$ & $€ /$ credito & $€$ \\
\hline \multicolumn{7}{|c|}{ Universidades públicas } \\
\hline$U D C$ & Presencial & 240 & 9,85 & $2.364,0$ & 9,85 & $2.364,0$ \\
\hline UEX & $\begin{array}{l}\text { Presencial } \\
\text { Online }\end{array}$ & 240 & 9,88 & $2.371,2$ & 10,07 & $2.416,8$ \\
\hline UGR & Presencial & 240 & 12,20 & $2.928,0$ & 12,49 & $2.997,6$ \\
\hline Unizar & Presencial & 240 & 12,58 & $3.019,2$ & 13,03 & $3.127,2$ \\
\hline UM & Presencial & 240 & 12,60 & $3.024,0$ & 14,09 & $3.381,6$ \\
\hline UV & Presencial & 240 & 12,11 & $2.906,4$ & 16,15 & $3.876,0$ \\
\hline Unileon & Semipresen. & 240 & 11,08 & $2.659,2$ & 16,67 & $4.000,8$ \\
\hline USAL & Presencial & 240 & 11,08 & $2.659,2$ & 16,67 & $4.000,8$ \\
\hline UC3M & $\begin{array}{l}\text { Presencial } \\
\text { Semipresen. }\end{array}$ & 240 & 14,05 & $3.372,0$ & 21,32 & $5.116,8$ \\
\hline UCM & Presencial & 240 & 14,05 & $3.372,0$ & 21,32 & $5.116,8$ \\
\hline$U B$ & $\begin{array}{l}\text { Presencial } \\
\text { Semipresen. }\end{array}$ & 240 & 15,16 & $3.638,4$ & 25,27 & $6.064,8$ \\
\hline \multicolumn{7}{|c|}{ Universidades privadas } \\
\hline UOC & Online & 240 & - & - & 50 & 12.000 \\
\hline
\end{tabular}

En cuanto al comportamiento de la matriculación de nuevo ingreso y el número de egresados, debe aclararse que no fue posible acceder a los datos completos de los masters oficiales identificados, ya que el INE (2012) desde el curso 2007/2008 los ofrece agrupados bajo las categorías de la Clasificación Nacional de la Educación (CNED). En consecuencia se analizaron (con otras fuentes) únicamente los datos correspondientes al número total de estudiantes matriculados en los 19 masters pertenecientes a las universidades que integran RUID. Se percibe una tendencia al alza para el período comprendido entre los cursos 2006/2007 y 2011/2012 que parece detenerse en el último curso (figura 1).

Igual que en el caso de los grados, los precios de los masters oficia-

tros o profesorado del área, observamos que los títulos que tienen tal vinculación son mayoritariamente los de temática generalista y gran parte de los de information management. Por otro lado, los títulos sobre museología y archivística son mayoritariamente ofertados por otros departamentos como historia del arte o centros privados, junto con algunos de gestión e incluso biblioteconomía impartidos por ingenierías.

Hay que destacar que el $51 \%$ de ellos son masters online y/o semipresenciales. Las universidades están apostando por esta modalidad (hace unos años era prácticamente inexistente), para poder llegar a sectores de población más diversos.

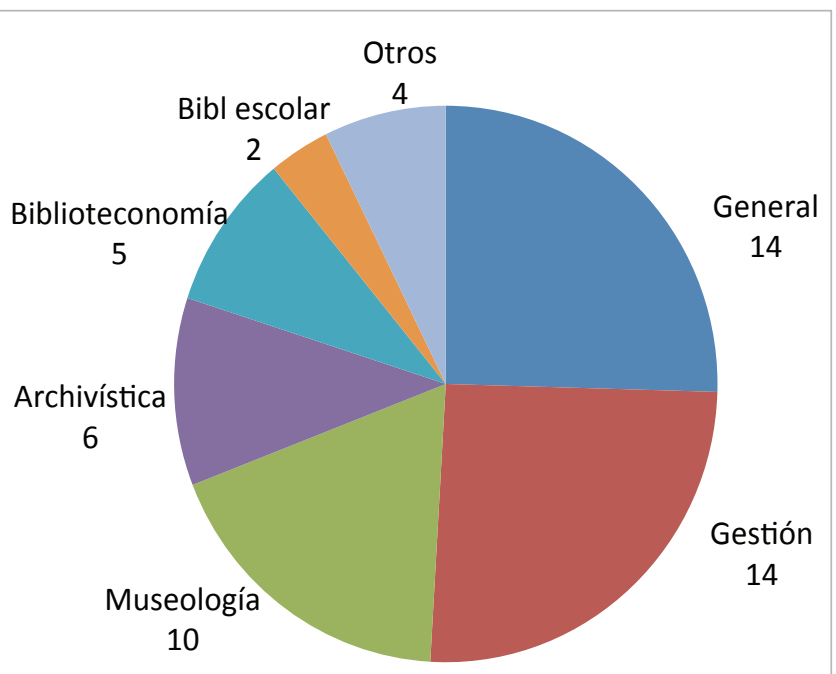

Figura 5. Distribución de titulaciones de segundo ciclo en el área, según cobertura temática principal. les varían sensiblemente como se muestra en la tabla 4, oscilando entre los 1.296 y los 4.246 anuales (60 ECTS).

La oferta de titulaciones de segundo ciclo con vinculación con el área es amplia, predominando los títulos generalistas de sesgo digital y los relacionados con information management

Si se comparan las subidas que han experimentado los precios de los estudios de grado y de master, resulta significativo el hecho de que las universidades madrileñas hayan encarecido en el primer caso más de un $34 \%$ y en el segundo más de un $60 \%$. La UB y USAL han subido más las tasas de grado que las de master. En cambio, comunidades como las de Andalucía, Extremadura y Aragón han apostado por el grado, encareciéndolo menos de un $5 \%$, mientras han aumentado los precios de las maestrías entre un 27 y un $62 \%$. Sólo la UDC ha mantenido el precio del grado, subiendo mínimamente el del master (6,2\%).

\section{Y ahora, tercer ciclo: doctorados}

En el curso 2012-2013 existen 13 programas de doctorado (tabla 3), regulados por distintos planes. Los del $R D$ 778/1998 están en extinción, siendo sustituidos por los del $R D$ 1393/2007, que a su vez han sido derogados o modificados en varios puntos por el $R D$ 99/2011. No obstante, tal y como plantea De-la-Moneda-Corrochano (2012) esta nueva ordenación propicia una distinción más clara entre los estudios de master y doctorado. Y se eliminan, también, las barreras entre masters de investigación y masters universitarios especializados o profesionalizantes. La tendencia, en la actualidad, es que se pueda acceder a los doctorados desde cualquier tipo de master oficial, acercándonos de esta forma a lo que ocurre en el resto del mundo. 
Tabla 3: Oferta de masters y doctorados (curso 2012-2013). Fuente: MEC (2013), RUID y sedes web de las universidades

\begin{tabular}{|c|c|c|c|}
\hline Institución & Título & ETCS & Mod \\
\hline \multicolumn{4}{|c|}{ Masters oficiales (Aneca) } \\
\hline \multicolumn{4}{|c|}{ Pertenecientes a departamentos de ByD } \\
\hline \multirow{3}{*}{ UAH } & Master en digital networked media and web science* & 60 & $\mathrm{O}$ \\
\hline & Master universitario en documentación & 60 & $\mathrm{~S}$ \\
\hline & Doctorado (con la Universidad Lusófona de Humanidades y Tecnologías de Lisboa) & & \\
\hline \multirow{2}{*}{ UB } & Master en bibliotecas y colecciones patrimoniales & 60 & $\mathrm{~s}$ \\
\hline & Doctorado & & \\
\hline$U B / U A B$ & Master en biblioteca escolar y promoción de la lectura & 60 & $\mathrm{~S}$ \\
\hline UB/UPF & Master en gestión de contenidos digitales & 90 & $\mathrm{P}$ \\
\hline \multirow{2}{*}{ UC3M } & Master universitario en bibliotecas y servicios de información digital & 60 & $\mathrm{~S}$ \\
\hline & Doctorado & & \\
\hline \multirow{3}{*}{ UCM } & Master en estudios avanzados de museos y de patrimonio histórico-artístico & 90 & $\mathrm{P}$ \\
\hline & Master en gestión de la documentación, bibliotecas y archivos & 60 & $\mathrm{P}$ \\
\hline & Doctorado & & \\
\hline \multirow{2}{*}{$U D C$} & Master universitario en ciencias documentales en el entorno digital & 60 & $\mathrm{~S}$ \\
\hline & Doctorado (con la UCM) & & \\
\hline \multirow{3}{*}{ UEX } & Master universitario en gestión de la información digital & 60 & $\mathrm{P}$ \\
\hline & Master universitario en investigación en ciencias sociales y jurídicas. Especialidad documentación y comunicación* & 60 & $\mathrm{P}$ \\
\hline & Doctorado & & \\
\hline \multirow{2}{*}{ UGR } & Master universitario en información y comunicación científica & 60 & $\mathrm{P}$ \\
\hline & Doctorado & & \\
\hline \multirow{2}{*}{ UM } & Master universitario en gestión de la información en las organizaciones* & 60 & $\mathrm{~S}$ \\
\hline & Doctorado & & \\
\hline Unileon & Doctorado & & \\
\hline \multirow{2}{*}{ Unizar } & Master en gestión de unidades y servicios de información y documentación (en extinción) & 120 & $\mathrm{P}$ \\
\hline & Doctorado & & \\
\hline \multirow{3}{*}{ UOC } & Master universitario en sociedad de la información y del conocimiento* & 60 & 0 \\
\hline & Master universitario en gestión estratégica de la información y el conocimiento & 60 & $\mathrm{O}$ \\
\hline & Doctorado & & \\
\hline UPF & Master universitario en documentación digital on line & 60 & 0 \\
\hline \multirow{2}{*}{ UPV } & Master universitario en contenidos y aspectos legales en la sociedad de la información & 75 & $\mathrm{P}$ \\
\hline & Doctorado & & \\
\hline \multirow{2}{*}{ USAL } & Master universitario en sistemas de información digital & 60 & $\mathrm{P}$ \\
\hline & Doctorado (en extinción) & & \\
\hline \multicolumn{4}{|c|}{ No pertenecientes a departamentos de ByD } \\
\hline \multirow{2}{*}{$U A B$} & Master oficial en archivistica y gestión de documentos & 120 & $\mathrm{P}$ \\
\hline & Master oficial en gestión de la información y el conocimiento en el ámbito de la salud & 60 & $\mathrm{P}$ \\
\hline$U M$ & Master universitario en educación y museos: patrimonio, identidad y mediación cultural & 60 & O \\
\hline \multirow{2}{*}{ UPV } & Master universitario en gestión de la información & 90 & $\mathrm{P}$ \\
\hline & Master en gestión de la innovación y el conocimiento & 60 & $\mathrm{P}$ \\
\hline US & Master en documentos y libros. Archivos y bibliotecas. & 60 & $\mathrm{P}$ \\
\hline UV & Master en sistemas y servicios en la sociedad de la información & 75 & $\mathrm{P}$ \\
\hline & $\begin{aligned} & \text { Títulos propios } \\
&\end{aligned}$ & & \\
\hline & Pertenecientes a departamentos de ByD & & \\
\hline$U R$ & Diploma de postgrado de librería & 30 & $\mathrm{P}$ \\
\hline UB & Master de gestión documental e información en las empresas & 72 & $\mathrm{~S} / \mathrm{O}$ \\
\hline$U B / U A B$ & Diploma de postgrado en bibliotecas escolares, cultura escrita y sociedad en red & 30 & $\mathrm{O}$ \\
\hline$U C 3 M / B N E$ & Master en bibliotecas y patrimonio documental & 60 & $\mathrm{O}$ \\
\hline UIJ & Postgrado en organización y gestión de la información & 22 & O \\
\hline & Posgrado en redes sociales e intercambio de conocimiento & 30 & $\mathrm{O}$ \\
\hline UOC & Diploma de posgrado en implantación de proyectos de gestión de documentos electrónicos & 30 & $\mathrm{O}$ \\
\hline UPF & Master en buscadores: marketing online, posicionamiento, community manager y content curator & 60 & O \\
\hline USAL & Master en edición & 60 & $\mathrm{P}$ \\
\hline & No pertenecientes a departamentos de ByD & & \\
\hline CEU & Curso superior de museología & 30 & $\mathrm{P}$ \\
\hline Deusto & Diploma de especialización en documentación y gestión del patrimonio histórico. Archivos, bibliotecas, museos & 48 & $\mathrm{P}$ \\
\hline & Master de biblioteconomía & 12 meses & $\mathrm{O}$ \\
\hline ETD & Master de archivística & 10 meses & 0 \\
\hline & Master de museología & 10 meses & $\mathrm{O}$ \\
\hline
\end{tabular}




\begin{tabular}{|c|c|c|c|}
\hline IIM & Master en museología & 12 meses & $\mathrm{O}$ \\
\hline$U A B$ & Diplomatura de postgrado en gestión, preservación y difusión de archivos fotográficos & 30 & $\mathrm{O}$ \\
\hline$U A H$ & Master en museología y museos & 60 & $\mathrm{O}$ \\
\hline UAM & Master en gestión de la documentación musical & 60 & $\mathrm{P}$ \\
\hline$U B$ & Master en gestión del patrimonio cultural & 90 & $\mathrm{P}$ \\
\hline \multirow{2}{*}{ UC3M } & Experto en community manager & 20 & $\mathrm{O}$ \\
\hline & Master en archivística & 90 & $\mathrm{O} / \mathrm{P}$ \\
\hline UC3M/URJC & Master interuniversitario en analista de inteligencia & 60 & $\mathrm{P}$ \\
\hline UCM & Master en social media y community manager & 60 & $\mathrm{P}$ \\
\hline UGR & Master en museología & 12 meses & $P$ \\
\hline UNED & Especialista universitario en archivística & 50 & $\mathrm{O}$ \\
\hline Unizar & Master en museos: educación y comunicación & 60 & $P$ \\
\hline UPF/MNAC & Programa de postgrado de la gestión museística: cómo hacer funcionar un museo & 30 & $\mathrm{P}$ \\
\hline
\end{tabular}

Nota: Los marcados con * corresponden a masters de investigación.

1 ETCS (European credit transfer and accumulation system) equivale a una carga de trabajo de 20-25h.

Mod = Modalidad: O: online, P: presencial, S: semipresencial

A pesar de todo, los datos disponibles sobre los doctorados en el área revelan un importante aumento del número de matriculados y tesis leídas, sobre todo a partir del curso 2007/2008 (figura 7). Debe señalarse que el INE (2012) no recoge datos de nuevo ingreso y que los datos de matriculación se facilitan agregados en las categorías CNED desde $2009 / 2010$. Por eso es difícil saber si pertenecen o no a programas del área.

Gran desigualdad en las tasas de matrícula entre las comunidades autónomas

En cuanto a las tesis leídas, los datos del INE y de Teseo (MEC, 2013b) no coinciden. Durante el período analizado, Teseo recoge un número mayor (254) que el registrado en el INE (192) (figura 7). La causa puede ser que Teseo utiliza descriptores múltiples y por tanto algunas no sean estrictamente del área, de ahí un número mayor. Y, sin embargo, el sistema de clasificación del INE es mucho más restringido. En este sentido hay que tener en cuenta que los doctorados se generalizan en los comienzos del siglo XXI. Hasta entonces, muchas tesis se leían en departamentos afines al área, pero eran dirigidas por doctores de departamentos de biblioteconomía. Aunque estos datos hay que tomarlos con precaución, y a pesar de la variabilidad de los mismos, la línea de tendencia es al alza; hecho que ya apuntaban para el 2008 Delgado-López-Cózar y
De-la-Moneda-Corrochano (2010).

Ninguno de los doctorados ha mantenido la mención de calidad en las nuevas normativas vigentes.

Los datos disponibles sobre los doctorados en el área revelan un importante aumento del número de matriculados y tesis leídas, sobre todo a partir del curso $2007 / 2008$

\section{Reflexiones}

Uno de los objetivos prioritarios de la implantación del grado de información y documentación fue adaptar los estudios a una nueva realidad de la profesión y del mercado de trabajo. Se trataba de dar respuesta a la caída de la demanda de la diplomatura y la licenciatura que ya habían detectado autores como Delgado-López-Cózar (2003; 2007), Delgado-López-Cózar y De-la-Moneda-Corrochano (2008; 2010), García-Marco (2009) entre otros. Por los datos analizados, podemos concluir que este objetivo no se ha conseguido. Aunque se ha remontado algo, la matrícula de nuevo ingreso lleva, de manera general, un camino descendente y las tasas de abandono, más altas de lo deseado, contribuyen a aumentar ese descenso en niveles de sostenibilidad.

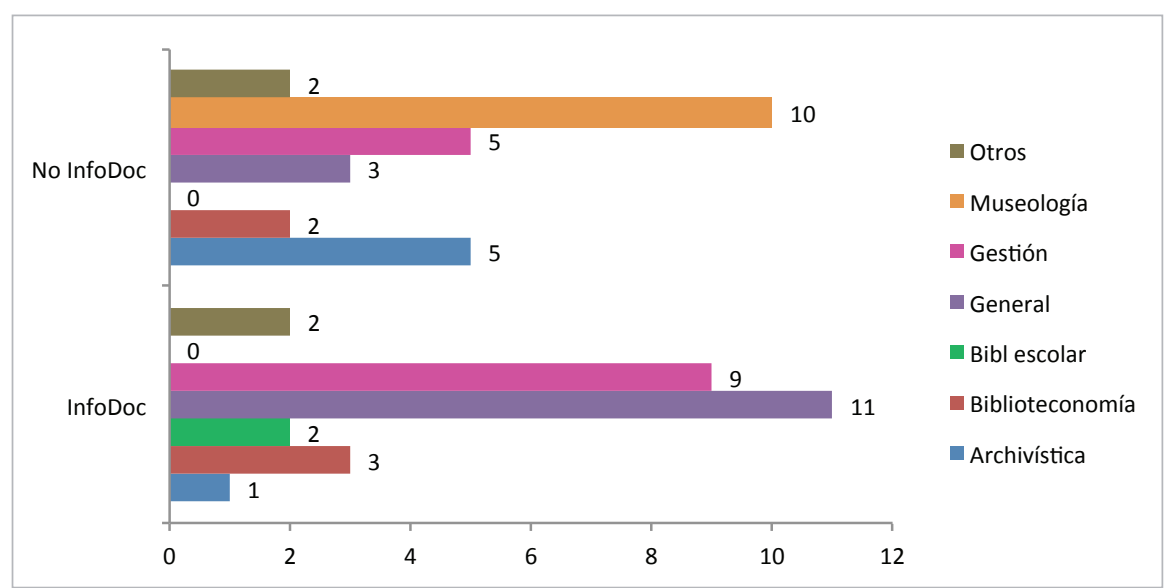

Figura 6. Títulos de segundo ciclo ofertados, por vinculación al área y cobertura temática principal
La escasa visibilidad social (ya endémica) de los estudios y de la profesión constituye una de las debilidades más importantes. No deja de ser paradójico que en una sociedad dominada por la información y por nuevos perfiles laborales ligados a ella, los grados no tengan más demanda.

Sin embargo, las matrículas de segundo y tercer ciclo (a pesar de la subida de tasas) se mantienen o incluso se incrementan desde 20072008, aunque esta tendencia parece detenerse en la actualidad y es difícil prever su evolución. Los nue- 
vos reales decretos han propiciado una mayor oferta de masters académicos de 60 ECTS y la extinción o transformación de muchos que eran sólo de investigación. Esto hace preguntarse, una vez más, si los estudios de documentación serían más apropiados para un segundo ciclo; algo que ya han planteado, también, otros autores como Delgado-López Cózar y De-la-Moneda Corrochano (2010) desde la disminución de la matrícula de la diplomatura y el aumento de la de la licenciatura que se inició en 20042005.

En la reunión de $R U I D$ se propuso un conjunto de medidas que pudieran contribuir a mejorar la situación, que resumimos a continuación.

- Acciones dirigidas a mejorar las enseñanzas impartidas: revisión y actualización de los planes de estudio de los grados en información y documentación, teniendo en cuenta las nuevas condiciones y enfoques profesionales en el mercado laboral. Para ello, convendría volver a abordar cuestiones como un análisis estratégico del mercado laboral y también del diseño e impacto de los estudios. Existen múltiples precedentes en nuestro país, muchos de ellos ya citados a lo largo del trabajo, y, en el ámbito internacional, algunos de los ejemplos más recientes son los resultados de la conferencia Information professionals 2050 (Marchionini; Moran, 2012) o el informe final del proyecto de las universidades australianas Re-conceptualising and re-positioning Australian library and information science education for the $21^{\text {st }}$ century (Partridge et al., 2011).

La escasa visibilidad social de la profesión constituye una de las debilidades más importantes de los estudios

- Revisión de la oferta de posgrados de especialización, dirigidos tanto a estudiantes del área como a profesionales en ejercicio (orientados a la actualización competencial y mejora de su desempeño laboral) y titulados de otras áreas, de corte técnico, cultural y científico.

- Realizar un mayor seguimiento y tutorización de los estudiantes a fin de detectar y prevenir el abandono.

- Establecimiento de alianzas estratégicas con otras áreas: colaboraciones curriculares, titulaciones conjuntas, etc.

- Marketing profesional en empresas: acciones encaminadas a incrementar la sensibilidad de las empresas hacia nuestra profesión.

- Acciones dirigidas a desarrollar la imagen de la profesión en el conjunto de la sociedad: aumentar la visibi-
Tabla 4: Precio de la primera matrícula completa de los masters oficiales (cursos 2011/2012 y 2012/2013). Fuente: MEC (2013a) y las sedes de las universidades

\begin{tabular}{|c|c|c|c|c|c|}
\hline \multirow{2}{*}{ Universidad } & \multicolumn{2}{|c|}{$2011 / 2012$} & \multicolumn{2}{|c|}{$2012 / 2013$} & \multirow{2}{*}{$\begin{array}{c}\text { Variación } \\
\text { (\%) }\end{array}$} \\
\hline & $€ /$ crédito & $\epsilon$ & $€ /$ crédito & $\epsilon$ & \\
\hline \multicolumn{6}{|c|}{ Oficiales (Aneca) } \\
\hline$U D C$ & 20,25 & 1.215 & 21,61 & 1.296 & 6,2 \\
\hline UEX & 16,30 & 978 & 22,37 & 1.342 & 27,1 \\
\hline USAL & 28,55 & 1.713 & 40,60 & 2.436 & 29,7 \\
\hline$U M$ & 23,01 & 1.380 & 40,61 & 2.436 & 43,3 \\
\hline$U B$ & 31,42 & 1.884 & 45,00 & 2.700 & 30,2 \\
\hline UGR & 28,60 & 1.716 & 60,00 & 3.600 & 52,3 \\
\hline$U A H$ & 25,89 & 1.553 & 65,00 & 3.900 & 60,2 \\
\hline UC3M & 25,89 & 1.553 & 65,00 & 3.900 & 60,2 \\
\hline UCM & 25,89 & 1.553 & 65,00 & 3.900 & 60,2 \\
\hline$U O C$ & - & - & 67,86 & 4.071 & - \\
\hline UPF & - & - & 79,00 & 4.740 & - \\
\hline UPV & 14,58 & 1.093 & 42,00 & 3.150 & 65,3 \\
\hline UPV & 14,58 & 1.312 & 42,00 & 3.780 & 65,3 \\
\hline Unizar & 18,45 & 2.214 & 49,33 & 5.919 & 62,6 \\
\hline$U A B$ & - & - & 70,77 & 8.492 & - \\
\hline
\end{tabular}

lidad en medios de comunicación, participación en eventos, artículos, entrevistas, etc.

- Acciones para aumentar la visibilidad en la Red, y particularmente en las redes sociales.

- Promoción conjunta universidades/asociaciones profesionales: portal informativo, vídeos, etc. En los centros de secundaria solemos ser unos desconocidos: el antiguo módulo de biblioteconomía hace tiempo que desapareció, lo que parece haber repercutido negativamente en el conocimiento de los estudios y de la profesión.

La situación actual debe preocupar (y ocupar) no sólo al entorno académico sino a la profesión en su conjunto. Es obvio que sin titulados no habrá profesionales, lo cual llevará a que la oferta del mercado se cubra, aún con más frecuencia que la actual, con perfiles poco adecuados.

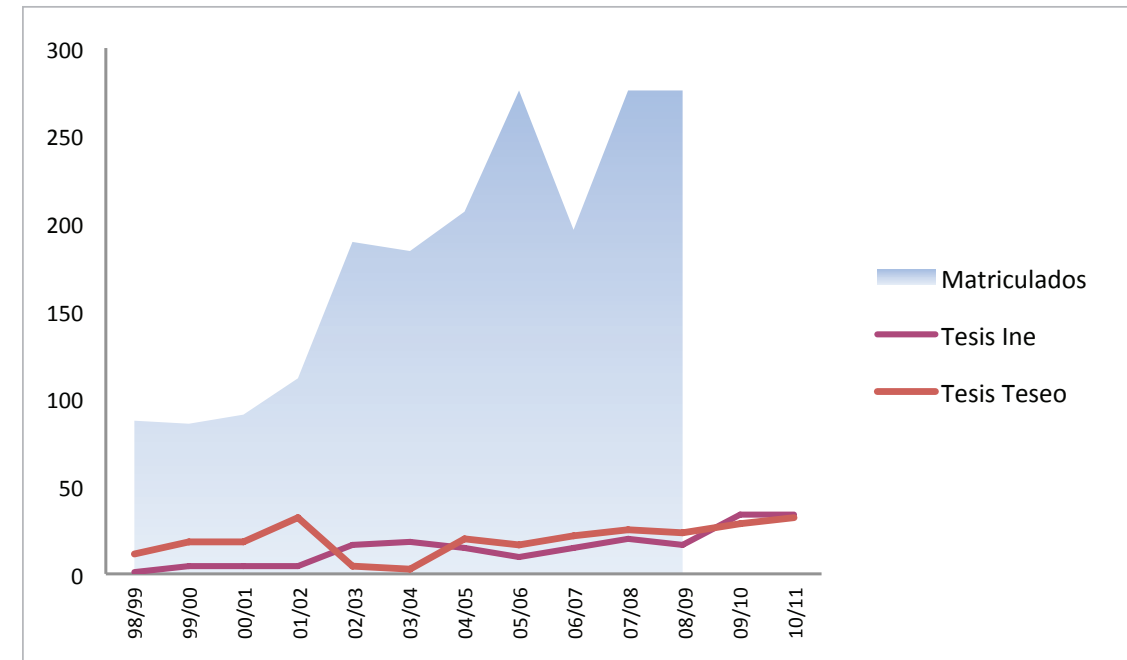

Figura 7. Evolución del número de matriculados en doctorado 1998/1999 a 2008/20098 y tesis leídas. Fuente: INE (2012, 2013), Teseo (MEC, 2013b) y universidades 


\section{Bibliografía}

Abadal, Ernest (1993). "La formación en biblioteconomía y documentación en España". Documentación de las ciencias de la información, n. 16, pp. 9-46.

http://revistas.ucm.es/index.php/DCIN/article/view/ DCIN9393110009A/20105

Abadal, Ernest; Miralpeix, Concepció (1999). “La enseñanza de la biblioteconomía y la documentación en la universidad española a finales de los noventa". BiD: Textos universitaris de biblioteconomia i documentació, marzo, n. 2.

http://www.ub.edu/bid/02abamir.htm

Abadal, Ernest; Borrego, Àngel; Serra-Pérez, Rafael (2012). "Mercado laboral de profesionales de la información: evolución de la oferta y de los perfiles ocupacionales". BiD: Textos universitaris de biblioteconomia i documentació, diciembre, n. 29.

http://www.ub.edu/bid/29/abadal2.htm

Agencia Nacional de Evaluación de la Calidad y Acreditación [España] (2004). Libro blanco: Título de grado en información y documentación. Madrid: Aneca.

http://www.aneca.es/var/media/150424/libroblanco_ jun05_documentacion.pdf

De-la-Moneda-Corrochano, Mercedes (2012). "Las cifras de la enseñanza universitaria en documentación en España: 2010". Anuario ThinkEPI, v. 6, pp. 13-30.

Delgado-López-Cózar, Emilio (2003). “Las cifras de la documentación en España: 2002". El profesional de la información, septiembre-octubre, v. 12, n. 5, pp. 344-367.

http://eprints.rclis.org/12858

Delgado-López-Cózar, Emilio (2007). “Las cifras de la infraestructura, profesión y educación documental en España: 2005". Anuario ThinkEPI, v. 1, pp. 32-42.

Delgado-López-Cózar, Emilio; De-la-Moneda-Corrochano, Mercedes (2008). "Las cifras de la enseñanza universitaria en documentación en España: 2006". El profesional de la información, julio-agosto, v. 17, n. 4, pp. 422-436.

http://dx.doi.org/10.3145/epi.2008.jul.10

Delgado-López-Cózar, Emilio; De-la-Moneda-Corrochano, Mercedes (2010). "Las cifras de la enseñanza universitaria en documentación en España: 2008". Anuario ThinkEPI 2010 , v. 4, pp. $26-40$

España. Ministerio de Educación, Cultura y Deporte (2013a). Estadísticas de precios públicos universitarios: Curso 2012-2013. http://www.mecd.gob.es/servicios-al-ciudadano-mecd/ estadisticas/educacion/universitaria/precios.html

España. Ministerio de Educación, Cultura y Deporte (2013b). Tesis doctorales: Teseo.

https://www.educacion.gob.es/teseo/irGestionarConsulta.do

Euroguide LIS: the guide to competencies for European professionals in library and information services (1999). European Council of Information Associations; Aslib y Leonardo da Vinci (Program). London: Aslib.

García-Marco, Francisco-Javier (2007). “Perspectivas sobre la enseñanza de posgrado en ciencias de la documentación en España". Ibersid, v. 1, pp. 291-302.

http://ibersid.eu/ojs/index.php/ibersid/article/view/3320

García-Marco, Francisco-Javier (2008). "La biblioteconomía y la documentación española en la encrucijada". Ibersid, v. 2, pp. 307-318.

http://ibersid.eu/ojs/index.php/ibersid/article/view/2254

García-Marco, Francisco-Javier (2009). “El movimiento ischools: posicionando los estudios de biblioteconomía y documentación en la era de la información". Anuario ThinkEPI, v. 3, pp. 95-99.

Hernández, Hilario (2001). “Qué bibliotecas públicas hay en el Estado español”. En: Las bibliotecas públicas en España: una realidad abierta. Madrid: Fundación Germán Sánchez Ruipérez.

Instituto Nacional de Estadística [España] (2012). Estadística de enseñanza universitaria: cursos 1998-1999 al 2010-2011. http://www.ine.es/jaxi/menu.do?type=pcaxis\&path=\%2Ft1 3\%2Fp405\&file $=$ inebase $\& L=0$

Instituto Nacional de Estadística [España] (2013). Anuario estadístico de España: Años 1983 al 1998.

http://www.ine.es/prodyser/pubweb/anuarios_mnu.htm

Marchionini, Gary; Moran, Barbara B. (eds.) (2012). Information professionals 2050: educational possibilities and pathways. North Carolina: School of Information and Library Science of University of North Carolina at Chapel Hill. ISBN: 9781300204862

http://sils.unc.edu/sites/default/files/news/InformationProfessionals-2050.pdf

Montes-López, Evelio (1995). “El mercado de trabajo de los documentalistas en España: análisis de una muestra de ofertas de empleo, 1984-1994". Revista española de documentación científica, v. 18, n. 2, pp. 178-187. http://dx.doi.org/10.3989/redc.1995.v18.i2.655

Morales-Sanabria, Carmen (2012). "Mapa de los estudios de información y documentación en España". Clip: boletín de Sedic, octubre-diciembre, n. 66.

http://www.sedic.es/p_boletinclip66_panorama_imprimir.htm

Partridge, Helen et al. (2011). Re-conceptualizing and repositioning Australian library and information science education for the $21^{\text {st }}$ century. Sidney: Australian Learning and Teaching Council. ISBN: 9780987198365

http://www.liseducation.org.au/resources/PP9-1326\%20 QUT\%20Partridge\%20Final_Report.pdf

Tejada-Artigas, Carlos; Moreiro-González, José-Antonio (2003). "Mercado de trabajo en biblioteconomía y documentación. Estudios sobre la inserción laboral de los titulados universitarios". El profesional de la información, enerofebrero, v. 12, n. 1, pp. 4-9.

http://dx.doi.org/10.1076/epri.12.1.4.19700

Tejada-Artigas, Carlos (2013). "Profesión y formación universitaria en 2012". Anuario ThinkEPI, v. 7, pp. 17-23. 